\title{
EL MALTRATO EN NIÑOS PREESCOLARES: ESTUDIO EMPÍRICO DE SUS CARACTERÍSTICAS PERSONALES Y FAMILIARES ${ }^{(*)}$
}

\author{
Estanislau Pastor Mallol, Teresa Zaplana Alcaraz \\ Sonia López Chivrall \\ Ángela Quintero Guevara, \\ Universidad "Rovira i Virgili" de Tarragona
}

\begin{abstract}
(*) La presente investigación ha sido posible gracias al convenio de colaboración suscrito entre la Universidad "Rovira i Virgili" de Tarragona y el EAIA Municipal de Tarragona del Departamento de Justicia de la Generalitat de Catalunya.
\end{abstract}

\section{RESUMEN}

Después de abarcar la perspectiva teórica del problema del maltrato infantil en el seno familiar, nuestro estudio pretende analizar las características personales y del entorno familiares de una muestra de niños y niñas entre 3 y 5 años de edad que se encuentran en el momento crítico de decidir si van o no a ser apartados de sus padres por los maltratos que han sufrido y determinar si dentro de este período de edad existen diferencias de género, y si éstas están dentro de un grupo determinado de edad.

La metodología utilizada se basa en evidencias obtenidas de casos registrados en sistemas legales y de salud. Nuestros sujetos son niños y niñas que tienen un expediente abierto en el EAIA (Equips d'Atenció a la Infància i Adolescencia) de Tarragona. El total de la muestra es de 21 niñas y 17 niños $(\mathrm{N}=38)$. El proceso seguido en el estudio tiene 5 fases: revisión bibliográfica, definición de variables, comprobación de calidad de los datos, análisis de los datos y discusión e interpretación de los resultados. Éstos se estructuran en dos apartados: resultados descriptivos referidos a la muestra y resultados obtenidos por tablas de contingencia.

Se destaca dentro de los resultados que el abandono no se relaciona con la edad de los padres ni con problemas de emigración. Pero en cambio, para casi la mitad de niños y niñas sus padres han sufrido maltrato familiar y la edad más problemática en la muestra ha sido los 4 años. 
PALABRAS CLAVE: Maltrato infantil, familia, preescolares.

\begin{abstract}
After discussing the theoretical framework of child abuse in families, our study aims to analyse the personal features and the family environment of a sample of children between 3 and 5 years old, who have reached the critical stage when it is necessary to decide whether they should be separated from their parents because of the abuse. The study also wishes to determine if there are gender differences at these ages and, if so, whether they occur at any particular age.

Our methodology uses evidence from cases registered in the legal and health systems. Our subjects are children who are being investigated by the EAIA (Caring Team for Childhood and Adolescence) in Tarragona. The total number of children in the sample is 21 girls and 17 boys $(\mathrm{N}=38)$. The instrument used to obtain data is the Evaluation Summary of Personal Record. The process used in the study has 5 steps: first, the bibliography was reviewed; then the variables were defined, the quality of the data tested and the data analysed; and, finally, the results were discussed and interpreted. The results are structured in two sections: the descriptive results of the sample and the results obtained from contingency tables.

It should be pointed out that parents do not abandon their children because of their age or because they emigrate. Rather, almost half of the parents whose children were in the study had suffered from similar abuse. Likewise, the most problematic age was 4 years old.
\end{abstract}

KEY WORDS: Infantile maltreatment, family, preschools.

\title{
INTRODUCCIÓN
}

No existe una única definición de maltrato infantil aceptada universalmente. Se trata de un término genérico que engloba diversas situaciones, que pueden ser vistas desde ópticas e intereses distintos.

En nuestra breve aproximación conceptual, nos centraremos en las definiciones propuestas por organismos nacionales e internacionales. La razón se encuentra en que dichas definiciones incluyen, amplían y/o complementan, tanto las clásicas de Kempe (1962) o de Wolfe (1998), como las más actuales de Roig y de Paúl (2000).

Para la Organización Mundial de la Salud (OMS) el abuso de menores abarca toda forma de maltrato físico y/o emocional, abuso sexual, abandono o trato negligente, explotación comercial o de otro tipo, de la que resulte un daño real o potencial para la salud, la supervivencia, el desarrollo o la dignidad del niño en el contexto de una relación de responsabilidad, confianza o poder. Según esta misma organización, se considera maltrato cualquier acto por acción u omisión realizado por individuos, por instituciones o por la sociedad en su conjunto y todos los estados derivados de estos actos o de su ausencia que priven a los niños de su libertad o de sus derechos correspondientes y/o que dificulten su óptimo desarrollo. 
La Comisión Nacional de Derechos Humanos (CNDH) enfatiza, en su definición de maltrato, sus efectos negativos para el niño: "Todo acto u omisión encaminado a hacer daño aún sin esta intención pero que perjudique el desarrollo normal del menor".

De estas y otras aproximaciones conceptuales del maltrato infantil podemos extraer algunas características comunes como son:

$\sqrt{ }$ Su carácter intencional, nunca accidental, del daño o de los actos de omisión llevados a cabo por los responsables del cuidado del niño(a), con el propósito de lastimarlo o injuriarlo o propinarle castigos inapropiados para su edad.

$\sqrt{ }$ Sus efectos negativos para la salud física y/o psíquica del niño y que comprometen su adecuado desarrollo, aunque provengan de actos de negligencia o incluso de omisiones.

$\sqrt{ }$ Sus efectos negativos aumentan en intensidad a medida que la situación se hace crónica o más severa.

$\sqrt{ }$ Su intensidad puede variar entre leve, moderada o grave y su ocurrencia puede ser antigua, reciente o recurrente.

$\sqrt{ }$ Abarca desde el período prenatal hasta la mayoría de edad.

Tras esta aproximación conceptual, nos vamos a referir a la magnitud del problema, haciendo especial hincapié en las edades en las que se produce el maltrato, el género de los sujetos afectados y, finalmente, las características de las propias familias.

Un primer dato que proviene de la Organización Mundial de la Salud (OMS) estima que 40 millones de niños son víctimas de la violencia en todo el mundo. Aunque no hay un registro oficial porque en general se trata de un "problema oculto".

Con suma frecuencia, detrás de problemas de comportamiento, de aprendizaje, etc. se esconden situaciones del maltrato, abuso y abandono en el pasado. Los daños emocionales causados al individuo en su edad más temprana pueden Ilegar a significar "marcas imborrables", que también se manifiestan en dificultades de ajuste personal y social. Las consecuencias del maltrato infantil se hacen visibles a medio y largo plazo en el desarrollo psicosocial y físico de los niños afectados.

Para determinar la incidencia del maltrato según las edades de los sujetos que lo sufren, una encuesta de la UNICEF (1999), basada en informes directos sobre el trato violento en el seno familiar, nos indica que el $28 \%$ de los menores de seis a nueve años, el 9\% de las niñas y niños de 10 a 13 años y el 10\% de los jóvenes de 14 a 17 fueron víctimas del mismo.

Desde una perspectiva histórica, la mayor parte de estudios coinciden en señalar que a las edades preescolares e incluso anteriores hay mayor frecuencia de violencia familiar. Los datos de la década de los 60 nos indican que muchos niños implicados en casos de maltrato tienen una edad inferior a los tres años de edad (Kempe y cols, 1962). En un estudio (Creighton,1989) con una muestra de 5708 casos de maltrato físico se puso de manifiesto que el 55\% niños tenía una edad inferior a los cuatro años, y que la variable edad fue inversamente proporcional al número de casos localizados, es decir, a mayor edad de los niños menor número de casos de maltrato. Finalmente, en la década de los noventa se encuentran datos que apoyan que el 94,3 \% de los niños maltratados tiene menos de cuatro años (Schloesser y cols.,1992). 
En un estudio sobre niños maltratados en Cataluña y que abarca del nacimiento a los18 años (Inglés, A. 2000), se afirma que, en relación con la población total, es a partir de los 4-5 años cuando se incrementa el porcentaje de niños maltratados. A los cuatro años se incrementan los malos tratos, pero en el escenario de un incremento de la población infantil en general.

En conclusión, aunque aquí hayamos aportado resultados de estudios que han utilizado metodologías distintas y efectuados en generaciones distintas, etc., se puede inferir que el maltrato infantil aparece predominantemente en las edades preescolares e incluso antes.

Por lo que respecta al género del niño maltratado, las investigaciones suelen confirmar que existe una mayor predisposición al maltrato de niños que de niñas (Gallardo y col, 1998). Desde una perspectiva que puede considerarse histórica, se nos afirma que ello puede deberse a que los niños son más desafiantes y responden a una agresión con otra, hecho que no suele ocurrir en las niñas, éstas suelen mostrar mayor inhibición y pasividad (Kinard, 1.978). No obstante, aportaciones algo más recientes apoyan que no existen diferencias significativas al comparar ambos géneros, con la excepción del abuso sexual que se da predominantemente en las niñas (Wolfe, 1998).

En el estudio citado anteriormente sobre niños maltratados en Cataluña (Inglès, A. 2000), se nos indica que el porcentaje de niños maltratados es superior que el de la niñas (54,1\% frente al 45,9\%). Los resultados correspondientes al año 1991 fueron muy similares (el $54,72 \%$ de niños frente a un $45,28 \%$ de niñas).

Si retomamos el estudio del año 2000 y nos limitamos a los datos referidos a las edades de 3 a 5 años, los resultados son los siguientes: En el intervalo de los 3-4 años, de un total de 306 sujetos que fueron objeto de maltrato, 172 fueron niños y 134 niñas. Esto representa el 3,9\% de niños y el 3\% de niñas del total de la población afectada. En el intervalo delos 4-5 años, de un total de 367 sujetos que fueron objeto de maltrato, 218 fueron niños y 149 niñas, lo que representa el 4,9\% de niños y el 3,4\% de niñas maltratadas del total de la población afectada.

Por lo que respecta al ámbito familiar y más concretamente a la problemática de la violencia intrafamiliar, es necesario abordarla desde diferentes perspectivas para poder garantizar una reflexión que nos permita comprender sus fuentes, controlar su frecuencia y, en último término, disminuir sus secuelas. Aunque no es objeto de este estudio, somos conscientes de que existen otros factores influyentes que no son exclusivamente los del núcleo familiar. De hecho, la permisividad y aceptabilidad del maltrato se encuentra en la estructura de la sociedad misma (Browwne y Falshaw, 2002).

Centrados en la familia, se hace indispensable justificar los orígenes de la violencia en alguna causa atribuible al propio núcleo familiar. Podemos destacar aquí que la causa de tal violencia suele explicarse por las deficiencias personales de los cuidadores intrafamiliares y en el estrés externo que afecta al núcleo familiar propiamente dicho (Barudy, 2000).

Se debe resaltar que el concepto de cuidador intrafamiliar surge de la existencia de dos tipos de roles a desempeñar, definidos de acuerdo con la función de satisfacción de necesidades básicas de carácter afectivo y económico que tiene la familia; estos roles son: el afectivo y el instrumental (Gracia y Musitu, 2001). El afectivo se relaciona con funciones de apoyo y de aportación de valores, normas y pautas de comportamiento en general y que suele ser desempeñado tradicionalmente por la 
madre. Por otro lado, el instrumental, que hace referencia a tareas que garantizan la supervivencia y el crecimiento y desarrollo familiar y, más específicamente, a la aportación de orden económico, que suele ser realizada tradicionalmente por el padre.

Sin embargo, la clase de cuidador intrafamiliar está también relacionada con el tipo de familia y consecuentemente su papel puede ser desempeñado, en muchos de los casos, por diferentes miembros e incluso por individuos de origen extrafamiliar.

Esto permite reconocer entonces factores de riesgo del menor y de la familia (Cantwell y Garbarino, 2003). Del primero se identifican como principales situaciones: deficiencia mental, parálisis, dificultades para la alimentación o de control de esfínteres, hiperactividad, hipoactividad, enfermedades crónicas, bajo rendimiento escolar, niños con autoestimas negativas, poco vínculo afectivo, rebeldía. Por lo que a las familias se refiere aparecen los factores de riesgo como: crisis familiares por necesidades básicas insatisfechas; pautas de crianza violentas; aceptación de la violencia como forma de relación; pobreza; padres adolescentes; baja escolaridad; hijos indeseados; disfunciones en la pareja; aislamiento social; baja escala de valores; consumo de sustancias psicoactivas; padres con enfermedades mentales; ausencia de una figura paterna; familias con antecedentes de abuso; hacinamiento; familias autoritarias; bajos niveles de comunicación; poca claridad en las normas; etc.

La puesta en marcha de soluciones a corto plazo para el cuidado de niños y de servicios de ayuda a los padres ha puesto de relieve que los malos tratos infantiles a menudo tienen lugar cuando los padres se encuentran bajo una fuerte y continua tensión producida por problemas familiares que no pueden controlar. Así como resultado de una cultura que aprueba el maltrato y lo legitima generacionalmente como una forma de mantener el control y la autoridad.

La preocupación generalizada, sobre todo en Occidente, ante el creciente número de denuncias de malos tratos infantiles ha llevado a aprobar leyes específicas que pretenden identificar, registrar y tratar este tipo de casos, aunque cada vez la atención está más enfocada a la prevención.

La historia de maltrato existente en las familias no es ajena al propio maltrato que los niños reciben. Los estudios han revelado que la mayor parte de los padres que abusan de sus hijos habían sufrido ellos también la misma situación por parte de sus progenitores. En la edad adulta las hijas de mujeres víctimas de la violencia doméstica tienen mayores probabilidades de sufrir ese tipo de agresiones, y los hijos de esas mujeres son más propensos a perpetrarla (Alvarado et al., 1998; Heise, 1994; Morrison y Orlando, 1999), probablemente por aprendizaje vicario.

Algunos investigadores afirman que este tipo de padres presentan una personalidad infantil, mientras que otros opinan que éstos esperan de forma poco realista que sus necesidades psicológicas sean cubiertas por sus hijos y que, al no ver cumplidas estas expectativas, experimentan un gran estrés y se vuelven violentos en las relaciones con ellos.

En una sociedad como la nuestra, en permanente cambio, el pretender abordar el estado actual del maltrato infantil y sus alcances, requiere de una revisión permanente de las variables implicadas, además de hacer uso de enfoques inductivos (Moreno 2002). El maltrato puede ser considerado como un síndrome que aparece como consecuencia de factores intrafamiliares originados por la incapacidad de los cuidadores de desempeñar normalmente su rol psicológico y personal. 
En el presente estudio hemos optado por este planteamiento inductivo, aunque ello no significa que, para obtener una explicación más global del problema, sea también necesario utilizar perspectivas deductivas, centradas en la influencia de factores extrafamiliares, es decir, las que se refieren a las causas generadas por el contexto social que rodea a la familia de estos niños.

Una vez analizada, desde una perspectiva teórica, la complejidad del maltrato infantil en el seno familiar, nos proponemos estudiar aquí las características personales y familiares de una muestra de niños y niñas de 3 a 5 años. Hemos elegido las edades preescolares por ser las que tienen una mayor incidencia por lo que al maltrato se refiere. Dentro del mismo período de edad, también nos interesa poder determinar si existen o no diferencias de género y si estas diferencias se dan dentro de un determinado intervalo de edad.

Hemos sesgado conscientemente la muestra para tener evidencias reales de maltrato a nivel familiar. Por esta razón la población de niños y niñas estudiados se encontraban en una situación crítica como es la de decidir si iban o no a ser apartados de sus padres a causa de los maltratos sufridos.

\section{MÉTODO}

\section{Sujetos}

La muestra está compuesta por 38 sujetos $(N=38), 21$ niñas $(55,26 \%)$ y 17 niños $(44,73 \%)$, con una edad comprendida entre 3 y 5 años y ha sido distribuida en dos grupos de edad:

- Grupo I: 14 niñas y 6 niños, de entre 3;0 y 4;6 años ( $n=20)$.

- Grupo II: 11 niños y 7 niñas, de entre 4;7 y 5;11 años ( $n=18)$.

Dicha muestra ha sido extraída del EAIA (Equips d'Atenció a la Infància i Adolescència) de Tarragona y recoge la totalidad de sujetos a los que se realizó una Síntesis Evaluativa entre los años 1991 a 2002. Se trata de niños y niñas que presentan una situación familiar problemática de riesgo a partir de la cual el EAIA ha tomado medidas administrativas y técnicas que pueden desencadenar un cambio de tutela.

\section{Instrumentos}

El instrumento principal de obtención de datos ha sido la Síntesis Evaluativa del expediente personal confeccionado por el EAIA para cada uno de los sujetos de la muestra. La Síntesis incluye información personal y familiar, obtenida de fuentes como: los propios profesionales del EAIA, el CSMIJ (Centre de Salut Mental InfantoJuvenil), el CAP (Centre d'Atenció Primària), y otros profesionales externos que han mantenido contacto directo con el sujeto.

Para el vaciado de los datos se elaboró un sistema de categorías "ad hoc". (Ver anexo 1)

El análisis cuantitativo se llevó a cabo mediante el programa informático SPSS versión 11.5 para Windows.

Todos los datos obtenidos han sido tratados con el máximo rigor y anonimato, con fines estrictamente científicos, de acuerdo con las normativas éticas que rigen los estudios psicológicos. 


\section{Procedimiento}

El proceso seguido en el presente estudio consta de las siguientes fases:

La primera fase consistió en una búsqueda bibliográfica de la literatura relevante sobre el tema objeto de estudio. Se abarcaron tanto aspectos teórico-conceptuales como los referidos a resultados provenientes de otras investigaciones.

En la segunda fase se definieron operativamente las variables incluidas en el estudio, relacionadas con los objetivos. Se elaboró un sistema de categorías para vaciar los datos/comportamientos que contenían los expedientes de los sujetos de la muestra.

En la tercera fase se comprobó la calidad de los datos a partir del cálculo de la concordancia entre e intraobservador obtenida mediante el coeficiente kappa.

En la fase cuarta se realizó el análisis de los datos mediante el paquete estadístico SPSS. Concretamente se elaboraron tablas de contingencia $2 \times 2$ para valorar la significación estadística al relacionar distintas variables.

Finalmente se procedió a la discusión e interpretación de los resultados.

\section{Resultados}

\section{Representación gráfica de algunos resultados descriptivos de la muestra}

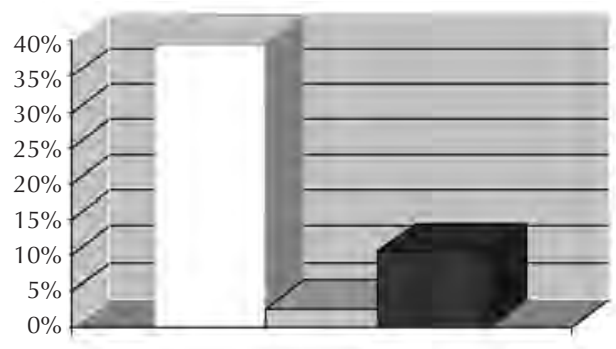

Figura 1: Información parto

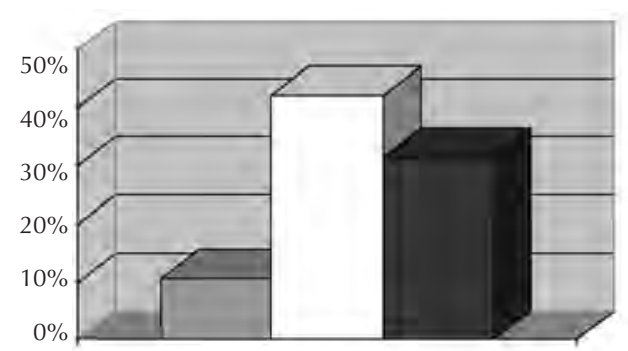

Figura 2: Retraso en el desarrollo

\section{bajo peso \\ $\square$ prematuridad \\ distócico}

口 carninar

$\square$ control efínteres

Elenguaje 


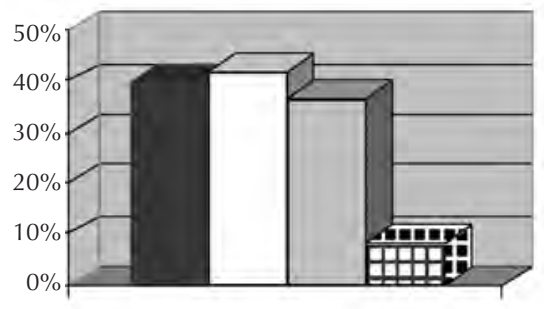

Figura 3: Conductas inadapatadas de interacción

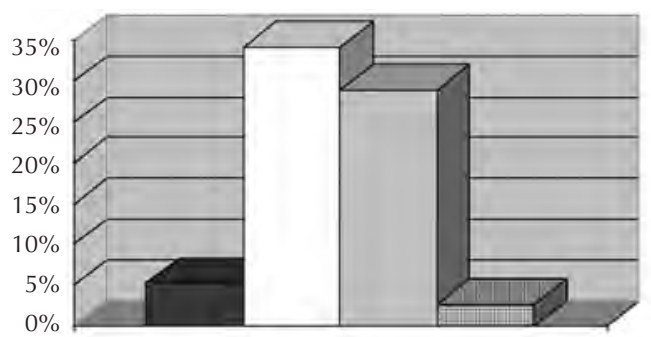

Figura 4: Conductas inadaptadas intrasujeto

\begin{tabular}{|l|}
\hline agresividad \\
$\square$ rebeldia \\
$\square$ celos \\
$\square$ mentiras \\
\hline
\end{tabular}

estereotipias $\square$ fobias y miedos $\square$ miedos nocturnos atics

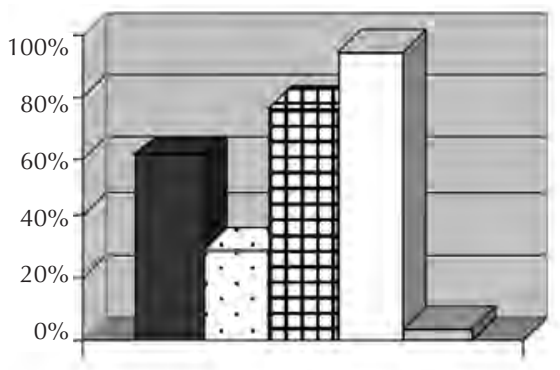

Figura 5: Tipos de maltrato

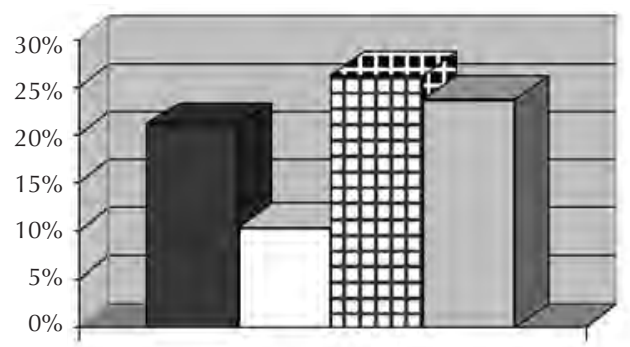

Figura 6: Dependencia de alguno de los padres

\section{m fisco}

$\square$ negligencia prenatal

प] negligencia postnatal

$\square$ emocional

G sexual

\begin{tabular}{l}
\hline alcohol padre \\
$\square$ alcohol madre \\
$\square$ drogodep.padre \\
$\square$ drogodep.madre
\end{tabular}

: alcohol padre $\square$ alcohol madre $\square$ drogodep.padre
$\square$ drogodep.madre 


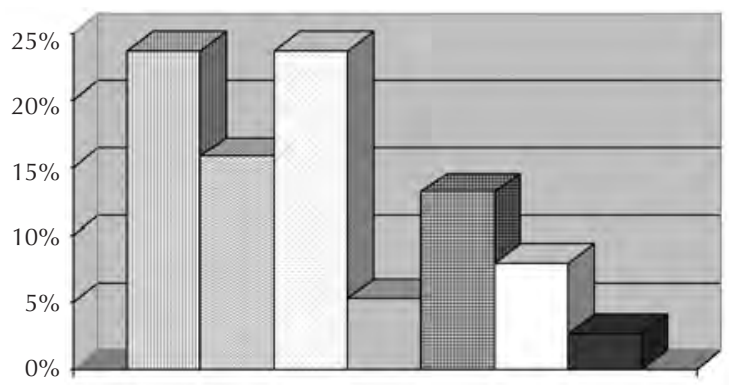

\begin{tabular}{|l}
$\square 2$ padres bio \\
$\square$ sólo madre \\
$\square$ madre + familia \\
$\square$ padre + abuela \\
⿴sólo abuela \\
$\square 2$ abuelos \\
$\square$ otros
\end{tabular}

Figura 7: Constelación familiar: cuidadores

Tabla I: Prueba de chi-cuadrado y niveles de significación de las tablas de contingencia.

\begin{tabular}{|lccc|}
\hline Variables & Valor & gl & Sig. asintótica (bilateral) \\
\hline Grupo edades * Sexo & 3,709 & 1 &, 054 \\
\hline $\begin{array}{l}\text { Grupo edades * Problemas mentales de alguno } \\
\text { de los padres (con o sin trastorno) }\end{array}$ & 3,767 & 1 &, 052 \\
\hline $\begin{array}{l}\text { Grupo edades * Funcionamiento familiar: } \\
\text { salvador }\end{array}$ &, 329 & 1 &, 045 \\
\hline $\begin{array}{l}\text { Edad * Padre y/o madre con VIH } \\
\text { Edad * Cuidador/a 1 y/o 2 con VIH del nucleo } \\
\text { familiar }\end{array}$ & 7,917 & 2 &, 002 \\
\hline $\begin{array}{l}\text { Edad * Rol asignado al cuidador/a principal: } \\
\text { salvador }\end{array}$ & 8,138 & 2 &, 017 \\
\hline $\begin{array}{l}\text { Sexo * Abandono de al menos uno de los } \\
\text { progenitores antes de los 5 años de edad del } \\
\text { niño/a }\end{array}$ & 4,354 & 1 &, 037 \\
\hline $\begin{array}{l}\text { Sexo * Historia del padre/madre de maltrato, } \\
\text { falta de afecto o desatención familiar en } \\
\text { su infancia }\end{array}$ & 3,823 & 1 &, 051 \\
\hline $\begin{array}{l}\text { Sexo * Alguno de los cuidadores del nucleo } \\
\text { familiar con historia de maltrato }\end{array}$ & 4,416 & 1 & 1 \\
\hline $\begin{array}{l}\text { Sexo * Sentimiento entre los cuidadores: } \\
\text { de afecto }\end{array}$ & 4,821 & & \\
\hline
\end{tabular}

(gl=grados de libertad; Sig.=significación) 


\section{Discusión /interpretación de los resultados}

Los resultados que vamos a presentar se estructuran en dos apartados:

En primer lugar se comentarán los resultados descriptivos de la muestra utilizada $y$, en segundo lugar, los resultados obtenidos a través de las tablas de contingencia con objeto de determinar la existencia de diferencias significativas entre las variables género y la edad de los sujetos de la muestra con respecto a las distintas variables estudiadas.

\section{Resultados descriptivos}

Los resultados los vamos a agrupar en tres apartados: variables del niño, variables relativas a los progenitores y hermanos biológicos y variables del núcleo familiar.

\section{Variables del niño}

\section{Información sobre la gestación y el parto:}

Por lo que respecta a la gestación y el parto, el 39,47\% de la muestra estudiada presentaba bajo peso al nacer y el 10,5\% sufrieron un parto distócico, siendo insignificante, en la población estudiada, la aparición de prematuridad en el parto (menos de 7 meses de gestación). (Ver figura 1).

\section{Retraso en el desarrollo:}

El 55,26\% de la muestra presentaban retraso en el desarrollo en al menos una de las tres áreas siguientes: motriz, cognitiva y madurativa. El 42,10\% de los sujetos presentaban retraso en el control de esfínteres y el 31,5\% tenían retraso en el desarrollo del lenguaje.

Por lo que se refiere al retraso locomotor, sólo un tanto por ciento menor de la muestra presentaba tales carencias (10,5\%) (Ver figura 2).

\section{Alteraciones del sueño y la alimentación en la primera infancia:}

El 52\% de la muestra presentaba alteraciones de la alimentación y un 39\% presentaba alteraciones del sueño.

\section{Conductas inadaptadas de interacción:}

Entendemos por conductas inadaptadas de interacción aquellas que se suelen manifestar en actos de rebeldía, agresividad, celotipias, mentiras y hurtos, fruto de relaciones interpersonales conflictivas.

Tomadas en su conjunto, el $71 \%$ de los sujetos de la muestra manifestaban algunas de las conductas antes mencionadas. De todas ellas la de mayor frecuencia de aparición es la rebeldía con un 42,1\%, seguida de conductas agresivas 39,5\% y celos $36,8 \%$. (Ver figura 3).

\section{Conductas inadaptas intrasujeto:}

Forman parte de conductas inadaptadas intrasujeto las estereotipias, fobias, miedos y tics. 
Agrupándolas, podemos decir que el $65,78 \%$ de los sujetos de la muestra las presentaban. Las más frecuentes fueron las conductas inadaptadas de fobias y miedos en la 1 a infancia $34,2 \%$ y miedos nocturnos $28,94 \%$. (Ver figura 4 ).

\section{Peso y talla inferiores:}

Más del $40 \%$ de los sujetos de la muestra presentaban peso y/o talla inferior al que les correspondía por edad. Concretamente un $47,36 \%$ bajo peso y un $44,73 \%$ baja talla.

\section{Tipos de maltrato:}

El $60,5 \%$ de los sujetos de la muestra presentaban maltratos físicos postnatales. El $78,94 \%$ padecían negligencia (28,94\% negligencia prenatal y 76,31\% negligencia posterior). El 94,73\% de los sujetos mostraban maltrato y abandono emocional. Un porcentaje insignificante, aunque no despreciable, 2,6\% sufrieron abuso sexual. (ver figura 5).

\section{Abandono de al menos uno de los progenitores antes de los 5 años de edad del niño/a: \\ El $57,89 \%$ de los sujetos de la muestra fueron abandonados por al menos uno de los progenitores (26,31\% por la madre y el 44,73\% por el padre) antes de los 5 años de edad. Los niños/as permanecieron separados de sus padres y/o madres una media de 2,5 años.}

\section{Variables relativas a los progenitores y hermanos biológicos}

\section{Edad padre/madre biológico/a:}

La media de edad del padre biológico de los sujetos de la muestra era de 35,40 años y la media de edad de la madre biológica era de 28,44 años.

\section{Años de emigración en Cataluña:}

El 92,1\% de los progenitores de la muestra eran emigrantes. La media de años que estas personas han vivido en Cataluña es de 21,4.

Historia del padre/madre de maltrato, falta de afecto o desatención familiar en su infancia:

El $42,1 \%$ de los progenitores (padres $18,42 \%$ y madres $36,84 \%$ ) de los niños que conforman la muestra presentaban historia de maltrato, falta de afecto o desatención familiar en su infancia.

\section{Encarcelamiento padre y/o madre:}

El 36,84\% de los progenitores de los niños de este estudio habían estado encarcelados (padres 34,21\%, madres 13,15\%, ambos 10,5\%). 


\section{Dependencia de alguno de los padres (alcoholismo y/o drogas):}

Más de la mitad de los padres, el 63,15\%, tenían dependencia al alcohol y/o drogas $(44,73 \%$ de los padres y $34,21 \%$ de las madres). (Ver figura 6$)$.

\section{Relación con la prostitución de alguno de los padres:}

El 36,84\% de los padres tenían relación con la prostitución.

\section{Algún hermano que haya estado en un CRAE (Centro Residencial de Acción Educativa ) o acogida familiar simple:}

La mayoría de los sujetos de la muestra, en concreto el 84,21\%, tenían algún hermano que estaba o había estado en un CRAE o en acogida familiar simple.

\section{Variables del núcleo familiar \\ Variables de constelación familiar:}

Más de la mitad de los niños de la muestra, 68,42\%, tenía como cuidadores al menos uno de los padres biológicos. Concretamente el 23,68\% de los niños tenían a ambos padres biológicos, el 39,47\% a la madre biológica y un porcentaje insignificante al padre biológico, 5,26\%. En cambio, un 31,58\% de los niños no tenía como cuidadores a ninguno de los padres biológicos. (Ver figura 7).

\section{Las características más importantes del núcleo familiar son las siguientes:}

En un $28,95 \%$ de los casos alguno de los cuidadores principales ha tenido historia de maltrato.

Han tenido dependencia a alcohol y/o drogas el 36,84\% de los cuidadores principales.

Finalmente han tenido relación con la prostitución el 18,42\% de los cuidadores principales.

\section{Dinámica familiar:}

En mayor medida, el rol asignado al cuidador/a/res principal/les es el afectivo con un $86,84 \%$ de los casos, seguido del rol ausente en un $36,84 \%$, el rol protector en un $26,32 \%$, los de autoridad y víctima en un $18,42 \%$ cada uno, el rol autoritario en un $13,16 \%$ y el rol salvador en un $10,53 \%$.

La relaciones intrafamiliares son violentas a nivel verbal en un 68,42\% de los casos y a nivel físico en un 57,89\%.

Los sentimiento entre los cuidadores son de afecto en un $39,47 \%$ y de hostilidad en un $50 \%$.

Los sentimiento entre los cuidadores y el sujeto de la muestra son de afecto en un $86,84 \%$ y de hostilidad en un $15,79 \%$.

Los sentimiento entre los hermanos y el sujeto de la muestra son de afecto en un $73,68 \%$ y de rivalidad u hostilidad en un $18,42 \%$.

Los sentimientos del sujeto de la muestra hacia sus hermanos son de afecto en un $78,95 \%$, y de rivalidad u hostilidad en un $21,05 \%$. 


\section{Resultados de las tablas de contingencias Comparaciones relativas al género}

En la muestra utilizada, si comparamos el grupo de niños con el de las niñas, podemos afirmar que de manera significativa (significación: 0.03) los niños han sufrido mayor abandono por parte al menos de uno de sus progenitores antes de cumplir los cinco años.

Por lo que se refiere a las historias de maltrato vividas por los progenitores de los niños y niñas de la muestra, es decir, falta de afecto o de desatención familiar, podemos afirmar que los padres/madres de las niñas estudiadas han sufrido mayor número de historias de maltrato (significación: 0.05), si las comparamos con las de los progenitores de los niños. No quiere decir que los padres y las madres de los niños de la muestra carecieran totalmente de este tipo de historias sino lo que ocurre que fue significativamente menor.

Este tipo de historia familiar más negativa en la muestra de las niñas, comparativamente con la de los niños (significación 0.03), también se repite cuando nos referimos a sus cuidadores y cuidadoras cuando éstos no son sus progenitores.

Un entorno familiar algo más positivo lo encontramos en la muestra de niños estudiados, al efectuar la comparación con las niñas del grupo (significación: 0.02). En el entorno familiar de los niños, sus cuidadores y cuidadoras han mantenido relaciones de afecto. No cabe duda de la importancia de estas relaciones ya que han podido beneficiar al niño o por lo menos aminorar, en parte, de forma directa y/o indirecta sus interacciones negativas anteriores. Al menos, en determinados momentos, han podido aprender de forma vicaria sobre las relaciones afectivas de sus cuidadores (modelos).

\section{Comparaciones relativas a la edad}

De los dos grupos de edad en los que se ha distribuido la muestra (3;0 a 4;6 años y 4;7 a 5;11 años), es el de mayor edad el que significativamente (significación: 0.05) tiene los progenitores con mayores índices trastorno mental. Problemática que puede estar o no acompañada de un trastorno específico manifiesto. Esto no significa que algunos de los progenitores del grupo de menor edad estén libres de dichos trastornos.

Si nos referimos a la edad de los sujetos en años cumplidos, son los niños y niñas de 4 años (significación: 0.002), comparados con los de 3 y 5 años, los que poseen un mayor número de progenitores portadores de inmunodeficiencia adquirida $(\mathrm{VIH})$, tanto si se trata de uno o de ambos progenitores. Esta significación también aparece (significación: 0.01) si nos referimos a los cuidadores de estos mismos niños y niñas de cuatro años cuándo sus cuidadores no son sus progenitores.

Si nos referimos a los roles que se suelen desempeñar en el contexto familiar, de todos los roles analizados sólo destaca el de "salvador". Su puesta en práctica de manera significativa por parte de los cuidadores aparece en el grupo de niños y niñas de 4 años (significación: 0.01), en contrate con las otras dos edades estudiadas.

Recordemos que por "salvador" se entiende a la persona que acoge, protege, etc. a los que no se valen por sí mismos. Esta acogida si se practica de una forma sana o realista es positiva, aunque se puede convertir en negativa cuando se ofrece una protección excesiva o innecesaria, impidiendo un desarrollo autónomo progresivo. De forma consciente o no los cuidadores pueden desempeñar este papel tratando de 
compensar lo que de negativo han vivido los niños y las niñas a lo largo de su historia personal.

\section{A modo de síntesis}

Vamos a proceder a detallar los aspectos más destacables de los preescolares estudiados, tanto los referidos a sus cuidadores familiares o no familiares como a ellos mismos.

En la muestra estudiada el abandono sufrido por los niños no se relaciona con la edad de los progenitores $y$, por tanto, con una posible inmadurez física, mental y/o emocional. Las edades promedio de los padres biológicos se sitúa entre los 30/40 años y de las madres en los 28. A partir de los datos tampoco se puede inferir que el problema de la emigración sea otro de los detonantes, añadidos a los problemas familiares, que incide en el maltrato, ya que se trata de personas que llevan por término medio mas de 20 años en Cataluña y, por lo tanto ya estables en su entorno.

Existen relaciones entre a las historias de maltrato de los progenitores y el maltrato real de los niños estudiados. Los progenitores de casi la mitad de los niños y niñas de la muestra han vivido maltrato familiar. Destacamos aquí la importancia que una historia de maltrato puede tener en un posterior maltrato de los hijos. No es anecdótico que la mayoría de los sujetos de la muestra tenga hermanos que también han sufrido algún tipo de maltrato y que han tenido que ser internados en un centro o familia de acogida.

A un nivel más global puede afirmarse que de manera significativa padres/madres y otros cuidadores /as de las niñas estudiadas han tenido una mayor historia de maltrato al compararlas con los niños. Podría hablarse en este caso de una historia de maltrato que se ha dilatado en el tiempo y ha pasado de padres y/o cuidadores/as a las hijas.

A esta historia de maltrato se le añade como característica destacable la drogodependencia (alcoholismo y/o drogadicción) de alguno de los progenitores y en menor grado la prostitución de la madre.

Un dato significativo que hemos encontrado se refiere a que tanto los progenitores como los cuidadores (uno o ambos) de los niños de cuatro años de la muestra estudiada poseen en mayor número el síndrome VIH.

Por lo que a nuestros niños y niñas se refiere, puede afirmarse que han sufrido mayoritariamente un abandono emocional. También aparecen conductas de negligencia y maltrato físico postnatal. Además, más de la mitad de la muestra ha sufrido un abandono total de al menos uno de sus progenitores antes de los cinco años de edad y esto acontece significativamente en los niños.

Con la pretensión de obtener una descripción del preescolar, por lo que al maltrato se refiere, y extrapolar determinadas acciones preventivas, podemos decir lo siguiente:

En nuestro estudio han sufrido maltrato de forma más intensa las niñas que los niños, resultados distintos a los obtenidos en otras investigaciones que afirman que son los niños los mayores receptores del maltrato (Gallardo y col, 1998; Inglès, A. 2000) o en otras donde no se encontrado diferencias entre géneros (Wolfe, 1988).

El perfil psicológico de los niños/as de la muestra nos indica que los sujetos implicados han presentado o están presentando un retraso en el desarrollo, alteraciones 
tanto en el sueño como en la alimentación y manifiestan conductas inadaptadas tanto a nivel individual (intrasujeto) como de interacción.

Si hemos de señalar cuál es la edad más problemática de maltrato dentro del período preescolar podemos afirmar que en la muestra estudiada ha sido la de los 4 años. Este resultado estaría en la línea de los datos aportados por el informe sobre el maltrato en Cataluña (Inglès, A. 2000) y otros resultados de estudios anteriores (Gill, 1.970), aunque se alejan de los obtenidos por otros autores (Schloesser y Cols.,1992).

Sería necesario contrastar las características de nuestra muestra con las muestras utilizadas en las investigaciones de los autores antes citados para poder justificar tales discrepancias. No obstante, queremos remarcar que los sujetos aquí estudiados habían pasado por situaciones severas de tipo familiar a partir de las cuales se debían tomar medidas que podían suponer la separación del niño de su entorno familiar. La homogeneidad de la muestra en este aspecto es importante ya que en otros estudios el control del maltrato puede quedar un tanto diluido. A todo ello se le pueden añadir los cambios generacionales de tipo socio-cultural a los que están sometidos dichas muestras y que en ocasiones impiden efectuar comparaciones debido a los sesgos introducidos.

\section{BIBLIOGRAFÍA}

Alvarado, G., Moysen, J., Estrada, S. \& Terrones, A. (1998). Prevalencia de violencia doméstica en la ciudad de Durango. Salud Publica de México 40 (6), 481-486.

Barudy, J. (2000). El dolor invisible de la infancia. Barcelona: Paidos.

Creighton, S. J. (1989). Fatal child abuse: How preventable is it? Child Abuse Review, 4, 318-328.

Garbarino, J., \& Cantwell. (2003). Psychological Maltreatment: Issues and Perspectives. En Steven, Kairys, Charles, Johnson. Psychological Maltreatment of Children and Youth. New York: Pergamon Press.

Gallardo, J. A., Trianes, M V. y otros. El maltrato físico hacia la infancia. (1.998). Málaga: Estudios y Ensayos. Universidad de Málaga.

De la Garza, J, Díaz, E, (1999). La violencia infantil. El abuso sexual y sus repercusiones en la salud pública. Salud Publica de México 3, 203-15.

Gill, D. (1970). Violence Against Children: Physical Child Abuse in the United States. Harvard: University Press.

Gracia, E. y Musitu, G. (2001). Psicología social de la familia. Barcelona: Paidos.

Herrada, A., Nazar, B., Pacher A, Cassaball, M., Vega, Ramos R. \& Nava, Cruz C. (1998). El niño maltratado en Tlaxcala: estudio de casos. Salud Publica de México, 34, 626-634.

Híjar Medina M., Tapia Yáñez R., López López M. V. \& Lozano, R . (1999) El trabajo materno y la gravedad de lesiones accidentales en niños. Salud Publica de México (3), 197-204.

Inglès, A. (2000). El maltractament d'infants a Catalunya. Quants. Com. Per què. Barcelona: Generalitat de Catalunya.

Kempe, H. C., Silverman, N. F., Steele, B. F., Droegemueller, W. \& Silver, H. K. (1962). The battered-child syndrome. JAMA. 181, 105-12. 
Kinard, E. M. (1978). Methodological issues and practical problems in conducting research on maltreated children. Child Abuse Neglect. 18, 645-656.

Morrison A, Orlando M. B. (1999). Social and economic costs of domestic violence: Chile and Nicaragua. Washington: Inter-American Development Bank, Johns Hopkins Press.

Moreno, J. M. (2002). Maltrato infantil. Madrid: Eos.

Roig, I., De Paul, J. (2000 ) Maltrato a los niños en la familia, evaluación y tratamiento. Madrid: Pirámide.

Schlösser, R., Hutchinson, M., Joseffer, S., Rusinek, H., Saarimaki, A., Stevenson, J., et al. (1992). Functional magnetic resonance imaging of human brain activity in a verbal fluency task. J Neurol Neurosurg Psychiatry, 64, 492-8.

Unicef (1999). Análisis de situación de menores en circunstancias especialmente difíciles. Madrid.

Wolfe, D. (1998). Programa de conducción de niños maltratados. México: Trillas.

\section{ANEXOS}

\section{Anexo 1: Definición de los tipos de maltrato utilizada en el estudio}

\section{- Maltrato físico prenatal:}

Se considera dicha situación cuando hay un consumo, sin control médico, por parte de la madre, de medicamentos, drogas o alcohol durante el embarazo, que provocan que el bebé nazca con un crecimiento anormal, patrones neurológicos anómalos, con síntomas de dependencia física de dichas sustancias, u otras alteraciones imputables a su consumo por parte de la madre.

La definición incluye también cualquier acto intencional producido por la madre del niño que implique o pudiera llevar consigo lesiones físicas (producidas con o sin instrumentos), enfermedades o intoxicaciones.

\section{- Negligencia prenatal:}

Entendemos por negligencia el dejar de hacer lo necesario para un adecuado desarrollo del feto, sea cualquier momento del período de gestación. Hace referencia a aquella situación intencional o no en la que la gestante no realiza los controles ni el seguimiento de su embarazo a cargo de un especialista.

\section{- Maltrato físico postnatal:}

Se define como cualquier acto producido en el niño, tras su nacimiento, que implique o pudiera llevar consigo lesiones físicas (producidas con o sin instrumentos), enfermedades o intoxicaciones.

Algunos indicios observables del maltrato físico posterior son: Hematomas, quemaduras, fracturas, u otras lesiones, mediante pinchazos, mordeduras, golpes, tirones de pelo, torceduras, quemaduras, puntapiés u otros medios con que se lastime el niño. La edad del menor, así como la forma y localización de dichas lesiones hacen muy improbable que tengan un origen accidental.

\section{- Negligencia postnatal:}

Hace referencia a aquella situación en la que las necesidades físicas básicas del menor no son atendidas temporal o permanentemente por ningún miembro 
adulto del grupo que convive con el niño/a, afectando la salud, la educación y el bienestar integral del menor ( Sullivan y Spacer, 1996 ). Tales necesidades físicas hacen referencia a las siguientes áreas: Alimentación, vestido, higiene, cuidados médicos, supervisión y vigilancia, condiciones higiénicas y seguridad en el hogar, y área educativa.

\title{
- Maltrato y abandono emocional:
}

En esta categoría se incluyen dos aspectos diferenciales: el maltrato y el abandono emocional.

Maltrato emocional: Se define como cualquier acto que rebaje la autoestima del niño o bloquee las iniciativas infantiles de interacción por parte de los miembros adultos del grupo familiar.

Puede presentar manifestaciones consecutivas a las conductas activas (rechazar, ignorar, aterrorizar, aislar) o bien de las conductas derivadas de la omisión (la privación de sentimientos de amor, afecto o seguridad, la indiferencia).

Se incluyen, por ejemplo: el rechazo verbal, la falta de comunicación, el insulto, la desvalorización repetida, la educación en la intimidación, la discriminación o las exigencias superiores a las propias de la edad o a sus capacidades.

\begin{abstract}
Abandono emocional: Situación en la que el niño no recibe el afecto, la estimulación, el apoyo y protección necesarios en cada estadio de su evolución y que inhibe su desarrollo óptimo. Existe una falta de respuesta por parte de los cuidadores a las expresiones emocionales del niño (llanto, sonrisa,...) o a sus intentos de aproximación o interacción. La conducta incluida en esta categoría es la indiferencia, que se refiere al estado de no disponibilidad de los adultos hacia el niño, es decir, el padre/madre/tutor, está preocupado por sí mismo y es incapaz de responder a la conducta y demanda del niño/a.
\end{abstract}

\section{- Abuso sexual:}

Hace referencia a cualquier clase de contacto sexual en un niño menor de dieciocho años por parte de un familiar/tutor adulto desde una posición de poder o de autoridad sobre el/la menor.

En este caso se consideran aquellas situaciones en los que el menor es utilizado por sus padres o tutores para realizar actos sexuales o como objeto de estimulación sexual. No es necesario que exista un contacto físico (en forma de penetración o tocamientos) para considerar que existe abuso sexual. Puede incluir desde la exposición de los genitales por parte del adulto hasta la violación del niño/a.

\section{Anexo 2: Información extraída de las síntesis evaluativas}

\section{DATOS DEL NIÑO/A:}

- Sexo

- Fecha de nacimiento

- Edad del niño/a cuando se abrió el expediente

- Edad del niño/a cuando se hizo la Síntesis 
Información sobre la gestación y el parto:

- Bajo peso al nacer: inferior a 2,5 Kilos

- Niño/a prematuro: duración de la gestación de 7 meses o inferior

- Parto distócico (o con problemas)

Retraso en el desarrollo:

- Retraso en el desarrollo del caminar

- Retraso en el desarrollo del control esfinteriano

- Retraso en el desarrollo del lenguaje

Alteraciones del sueño y la alimentación en la primera infancia:

- Alteraciones del sueño en la primera infancia

- Alteraciones de la alimentación en la primera infancia

Conductas inadaptadas de interacción en la primera infancia:

- Conductas inadaptadas de Agresividad en la primera infancia

- Conductas inadaptadas de Rebeldía en la primera infancia

- Conductas inadaptadas de Celos en la primera infancia

- Conductas inadaptadas de Mentiras en la primera infancia

- Conductas inadaptadas de Robos en la primera infancia

Conductas inadaptadas intrasujeto en la primera infancia:

- Conductas inadaptadas de Hiperactividad o pasividad en la primera infancia

- Conductas inadaptadas de Estereotipias en la primera infancia

- Conductas inadaptadas de Fobias y miedos en la primera infancia

- Conductas inadaptadas de Miedos nocturnos en la primera infancia

- Conductas inadaptadas de Tics en la primera infancia

Retraso mental:

- Retraso mental según ICAS (indicar el \%)

- Retraso mental según Raven (indicar el percentil)

Situación de abandono o defunción parental en los primeros años de vida del niño:

- Abandono de la madre antes de los 5 años de edad del niño/a

- Abandono del padre antes de los 5 años de edad del niño/a

- Defunción de la madre antes de los 5 años de edad del niño/a

- Defunción del padre antes de los 5 años de edad del niño/a

Otros datos del niño/a:

- Secuelas físicas importantes en el sujeto por causas múltiples (enfermedad, accidente, etc.

- Síndrome de abstinencia

- Seropositivo

- Peso en la síntesis: Normal/Inferior/Superior (según tabla...)

- Talla en la síntesis: Normal/Inferior/Superior 


\section{DATOS DE LOS PROGENITORES BIOLÓGICOS:}

(Información recogida sobre la madre y sobre el padre)

- Edad

- Fecha nacimiento

- Años de emigración en Cataluña

- Años que el niño/a ha permanecido separado del progenitor/a

- Historia del progenitor/a de maltrato, falta de afecto o desatención familiar en su infancia

- Encarcelamiento del progenitor/a

- Internamiento en psiquiátrico del progenitor/a

- Trastorno psiquiátrico del progenitor/a

- Progenitor/a con VIH

- Alcoholismo del progenitor/a

- Drogodependencia del progenitor/a

- Padre proxeneta

- Prostitución madre

- Retraso mental progenitor/a

- Exclusión social de los padres

\section{DATOS DE LA CONSTELACIÓN FAMILIAR:}

- Ve al padre y/o madre biológicos una vez al año como mínimo

- Familia monoparental

Quienes son los cuidadores:

- Padre biológico

- Madre biológica

- Padres no biológicos

- Acompañante padre o madre

- Abuelo/a

- Tío/a

- Cuidadores: Otros (CRAE, etc.)

Datos sobre los hermanos/as del niño/a:

- Número de hermanos en el núcleo familiar

- Número de hermanas en el núcleo familiar

- Número de hermanos/as en adopción

- Posición que ocupa el niño

- Algún hermano con medidas judiciales o administrativas

- Algún hermano que haya estado en CRAE o acogida familiar simple 


\section{DATOS DEL NÚCLEO FAMILIAR:}

(Información recogida de los dos cuidadores principales )

- Años de emigración en Cataluña

- Historia sufrida de maltrato, falta de afecto o desatención familiar en su infancia

- Encarcelamiento del cuidador/a

- Internamiento en psiquiátrico del cuidador/a

- Trastorno psiquiátrico del cuidador/a

- Cuidador/a con VIH

- Alcoholismo del cuidador/a

- Drogodependencia del cuidador/a

- Cuidador proxeneta o Prostitución de la cuidadora

- Retraso mental cuidador/a

- Exclusión social de los cuidadores

\section{DATOS DE LA DINÁMICA FAMILIAR:}

- Rol asignado al cuidador/a/res principal/les:

- Autoritario: quien sin más impone y exige a los demás su voluntad.

- De autoridad: persona que establece los parámetros normativos de actuación en la casa, p.ej. la hora de dormir, de comer, etc.

- Afectivo: quien muestra más afectos hacia el niño/a.

- Chivo expiatorio: quien carga con las culpas de todo.

- Víctima: persona que cree que todo (culpas, responsabilidades) recae sobre él.

- Protector: quien cubre las necesidades materiales del niño/a.

- Salvador: persona que acoge a los que no se valen por sí mismos, desde su criterio.

- Perseguidor: quien critica y saca defectos a los otros.

- Ausente: persona que no participa en la dinámica familiar.

Relaciones intrafamiliares violentas:

- de tipo verbal

- de tipo físico

Sentimientos entre los cuidadores:

- de afecto

- de rivalidad

- de hostilidad

Sentimientos entre cuidadores y niño/a:

- de afecto

- de rivalidad

- de hostilidad 
EL MALTRATO EN NIÑOS PREESCOLARES: ESTUDIO EMPÍRICO DE SUS CARACTERÍSTICAS PERSONALES Y FAMILIARES

Sentimientos entre los hermanos/as y el niño/a:

- de afecto

- de rivalidad

- de hostilidad

Sentimientos del niño/a hacia sus hermanos/as:

- de afecto

- de rivalidad

- de hostilidad 